\title{
Minimum Cost Path Algorithm for Coronary Artery Central Axis Tracking in CT Images
}

\author{
S.D. Olabarriaga ${ }^{1}$, M. Breeuwer ${ }^{2}$, and W.J. Niessen ${ }^{1}$ \\ 1 University Medical Center Utrecht, Image Sciences Institute, \\ Heidelberglaan 100, 3584 CX Utrecht, NL \{silvia,wiro\}@isi.uu.nl \\ 2 Philips Medical Systems, Medical IT - Advanced Development, \\ Building QV 162, P.O. Box 10000, 5680 DA Best, NL \\ marcel.breeuwer@philips.com
}

\begin{abstract}
The quality of cardiac images acquired with multi-detector CT scanners has improved significantly, to the point where minimally invasive examination of the coronary arteries became reality. The interpretation of such images requires efficient post-processing tools to isolate the vessels from other structures, such that they can be properly analyzed quantitatively or visually. In this paper we evaluate a method for semiautomated extraction of the central axis of coronary arteries in these images. First the vessels are enhanced with a local filter that analyzes the main modes of second-order variation in image intensity to determine the type of local structure. Secondly, the extremities of the axis are indicated by the user. Finally, a connected path between the given points is automatically determined with a minimum cost path algorithm, where the cost corresponds to the reciprocal of the enhanced image. The results obtained with different vessel enhancement filters are compared with manually traced axes in the evaluation of 15 cases.
\end{abstract}

\section{Introduction}

The latest developments in multi-detector CT and reconstruction techniques allow for fast acquisition of high-resolution images, opening up new possibilities in medical imaging. In particular, the promise of assessment of coronary artery disease in a less invasive manner than coronary angiography has created great expectation in health care [1]. Cardiac multi-detector CT (CMDCT) images are scanned after bolus injection (for blood enhancement), with high in-plane resolution and small slice spacing (order of $0.4 \mathrm{~mm}$, isotropic voxels). ECGgated retrospective reconstruction is used for improved temporal resolution. The resulting images contain much detail of the complex heart structure, allowing for the inspection of not only the coronary arteries, but also the heart chambers, pulmonary veins and related structures. Due to the amount of detail contained in CMDCT images, it is difficult to interpret them for coronary artery (CA) assessment. CAs are narrow tubular structures with tortuous trajectories around and between the heart chambers, therefore only small stretches can be visualized on a single plane (see Fig. [1). Post-processing tools are needed to facilitate their 
inspection, since it seems unlikely that a proper analysis can be performed in the usual slice-by-slice manner. For example, cross-sectional and longitudinal views allow for vessel analysis using the vessel itself as a reference (see Fig. 1). These views are generated using a description of the central vessel axis (CVA). A number of other tasks can also be facilitated when the CVA is known, such as initializing a wall segmentation method [2], or stenosis quantification. Usually the CVA is obtained manually by indicating points along the vessel path, a task that demands much time, and therefore is not suitable for daily practice.
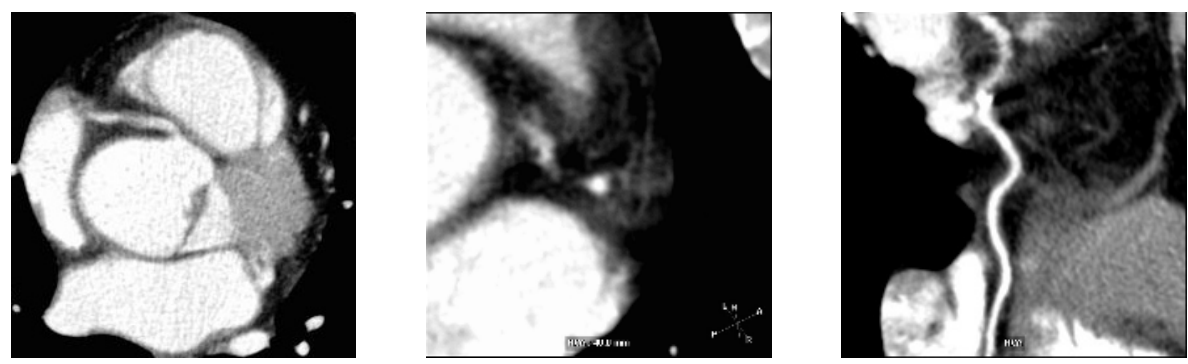

Fig. 1. Illustration of a coronary artery segment in a CMDCT image: in a slice (left), cross section (center) and longitudinal cut along the vessel (right).

Several automated methods for the extraction of the central axis of tubular structures have been described in the literature (e.g., [3], [4], 5] [6]). Our study started by trying to apply one of these methods, namely that proposed by Wink et al. in [3], to extract the CVA of coronary artery segments (CAS) in CMDCT images. In this approach, the vessels are initially enhanced with a local filter (Sect. 2), and the reciprocal of the enhanced image is used as cost function to determine a connected path between two given points with the minimum cost path algorithm (Sect. 3). Our motivation to apply this method for CMDCT images resides on the fact that this method is simple, fast, and has been sucessfully adopted in other imaging modalities and vessel types (MR, coronaries [3], MR carotids [2], MR, aorta [5]). In this study, however, we introduce a minor modification to the method described in [3]: instead of using the vessel enhancement filter proposed by Frangi et al. 7], we also investigated other filters, namely those proposed in [8] and [9]. The paths obtained with a number of CVA enhancement options are evaluated and compared based on reference paths traced manually (Sect. 4). Results as presented in Sect. [5 and discussed in Sect. 6]

\section{CVA Enhancement with Hessian-Based Filters}

The vessels are enhanced with filters that extract information from $2^{\text {nd }}$-order derivatives at multiple scales to identify the type of local structure in the image 
(e.g. tubular-like, sheet-like, blob-like). This is achieved by inspecting the main modes of variation in the Hessian matrix using eigenvalue decomposition. In this study we considered the Hessian-based filters (HBVF) proposed by Lorenz et al. [8], Sato et al. 9], and Frangi et al. [7.

In a multi-scale framework, a HBVF is defined as

$$
F(\mathbf{x})=\max _{\sigma} f(\mathbf{x}, \sigma),
$$

where $\mathbf{x}$ is a position in the image, $f$ is the filter, and $\sigma$ is the scale for calculating Gaussian image derivatives. The filters calculate $2^{\text {nd }}$-order derivatives, build the Hessian matrix $\mathcal{H}$, decompose it into eigenvalues $\lambda_{1}, \lambda_{2}$ and $\lambda_{3}$, and analyze them to determine the likelihood of $\mathbf{x}$ belonging to a vessel. This analysis is based on the following hypotheses (for bright vessels, dark background, and eigenvalues ordered as $\left|\lambda_{1}\right|<\left|\lambda_{2}\right|<\left|\lambda_{3}\right|$ ): (a) $\lambda_{1} \approx 0$ corresponds to the eigenvector tangent to the CVA; (b) $\lambda_{2} \approx \lambda_{3}<0$; and (c) $\left|\lambda_{1}\right| \ll\left|\lambda_{2}\right|$. The filters differ in how they test the hypotheses and generate a quantity indicating vessel-likelihood. In all cases, $f(\mathbf{x}, \sigma)=0$ if $\lambda_{2}>0$ or $\lambda_{3}>0$.

The filter defined by Lorenz et al. 8] was implemented as:

$$
\mathcal{L}(\mathbf{x})=\sigma^{\eta}\left|\frac{\lambda_{2}+\lambda_{3}}{2.0}\right|,
$$

where $\eta$ normalizes responses across scales.

The filter defined by Sato et al. in [9] was implemented as follows:

$$
\mathcal{S}(\mathbf{x})= \begin{cases}\sigma^{2}\left|\lambda_{3}\right|\left(\frac{\lambda_{2}}{\lambda_{3}}\right)^{\xi}\left(1+\frac{\lambda_{1}}{\left|\lambda_{2}\right|}\right)^{\tau}, \quad \lambda_{3}<\lambda_{2}<\lambda_{1}<0 \\ \sigma^{2}\left|\lambda_{3}\right|\left(\frac{\lambda_{2}}{\lambda_{3}}\right)^{\xi}\left(1-\rho \frac{\lambda_{1}}{\left|\lambda_{2}\right|}\right)^{\tau}, \quad \lambda_{3}<\lambda_{2}<0<\lambda_{1}<\frac{\left|\lambda_{2}\right|}{\rho}\end{cases}
$$

where $\xi \geq 0$ controls cross-section asymmetry, $\tau \geq 0$ controls the sensitivity to blob-like structures, $0<\rho \leq 1.0$ controls sensitivity to the vessel curvature, and $\sigma^{2}$ normalizes responses across scales.

The filter defined by Frangi et al. in [7] was implemented as follows:

$$
\mathcal{F}(\mathbf{x})=\left(1-e^{-\frac{A^{2}}{2 \alpha^{2}}}\right) e^{-\frac{B^{2}}{2 \beta^{2}}}\left(1-e^{-\frac{S^{2}}{2 \gamma^{2}}}\right)
$$

where $A=\frac{\left|\lambda_{2}\right|}{\left|\lambda_{3}\right|}$ (controlled by $\alpha$ ) discriminates plate- from line-like structures; $B=\frac{\left|\lambda_{1}\right|}{\sqrt{\left|\lambda_{2} \lambda_{3}\right|}}($ controlled by $\beta$ ) discriminates from blob-like structures and $S=\sqrt{\lambda_{1}{ }^{2}+\lambda_{2}{ }^{2}+\lambda_{3}{ }^{2}}$ (controlled by $\gamma$ ) eliminates background noise. Scale normalization is achieved by multiplying $\mathcal{H}$ by $\sigma^{2}$ before eigenvalue decomposition.

In this study, the filter response was calculated for 10 exponentiallydistributed scales in the interval $[0.5,5] \mathrm{mm}$. The parameter configuration for each filter was determined in the study reported in [10], namely: Lorenz-Eq.(2): $\eta=0$, Sato-Eq.(3): $\rho=1, \tau=0.5, \xi=1$, Frangi-Eq.(4): $\alpha=1, \beta=0.1$, $\gamma=100$. In the average, these configurations provided best CVA enhancements for the CAS considered here. 


\section{Minimum Cost Path Search}

In [6], the problem of CVA tracking is formulated as finding a connected path between two given points $\mathbf{a}$ and $\mathbf{b}$ in a cost image such that the total cost along the path is minimum. Different search strategies are discussed in [6], e.g., unidirectional (expand the search front from $\mathbf{a}$ until it reaches $\mathbf{b}$ ) or birectional (simultaneously expand from $\mathbf{a}$ and $\mathbf{b}$ until the search fronts meet). In this study we adopted bidirectional search because it is faster and provides the same results as with unidirectional search.

The cost image in this case is the reciprocal of the vessel-enhanced image, calculated as

$$
C(\mathbf{x})= \begin{cases}F(\mathbf{x})^{-n}, & F(\mathbf{x})<=T \\ M, & \text { otherwise }\end{cases}
$$

where $T$ is a threshold indicating a lower bound for vessel enhancement, and $M$ is the cost given to non-vessel positions. We found that results are quite insensitive to the values of $n$ and $T$ when the CVA enhancement is good; the adopted values are $n=1, M=10^{5}$ and $T=M^{-\frac{1}{n}}$. Note: the search is restricted to a bounding box determined from a dilated version of the CVA traced manually (diameter $\approx 10 \mathrm{~mm}$ ). Prior to cost computation, the values of $F(\mathbf{x})$ are normalized into the $[0,1]$ range with respect to the highest response within the bounding box.

The initial and final positions used to initialize the MCP method correspond to the first and last points in the manually traced CVA. The resulting path was exported as a chain of voxel positions, which were then compared with the manual CVA.

\section{Evaluation Strategy}

Fifteen coronary artery segments (CAS) in 5 CMDCT images acquired with 4-detector Philips CT scanners at different sites were used (spacing: in-plane $\in[0.4,0.55] \mathrm{mm}$, slice $\in[0.6,1.3] \mathrm{mm})$. The images were resampled to obtain isotropic voxels. The CVA of $15 \mathrm{CAS}$, with lengths $\in[17,115] \mathrm{mm}$, were traced manually by one operator using the Path Tracking Tool of the Philips EasyVision workstation. These curves were compared to the CVA determined automatically by the MCP method using cost functions based on different HBVF. Results are evaluated based on an error measure corresponding to the distance $D_{A R}(t)$ between all points in the automatic path $A(t)$ and the reference path $R(s)$ computed as follows:

$$
D_{A R}(t)=\min _{s}|A(t)-R(s)|,
$$

where $t$ and $s$ are path positions. The mean distance $\mu_{D}$ is used for general quality assessment:

$$
\mu_{D}=\frac{1}{n} \sum_{i=1}^{n} D_{A R}\left(t_{i}\right)
$$

where $n$ is the number of points along the discrete path. 


\section{Results}

A summary of the results obtained in this study is presented in Table 1 .

Table 1. Distance from $A$ to $R$ for cost functions based on CVA enhancement with the Lorenz, Sato and Frangi filters (see Sect. 2]: length ( $\mathrm{mm})$, minimum and maximum values, mean $\left(\mu_{D}\right)$ and standard deviation, in $\mathrm{mm}$.

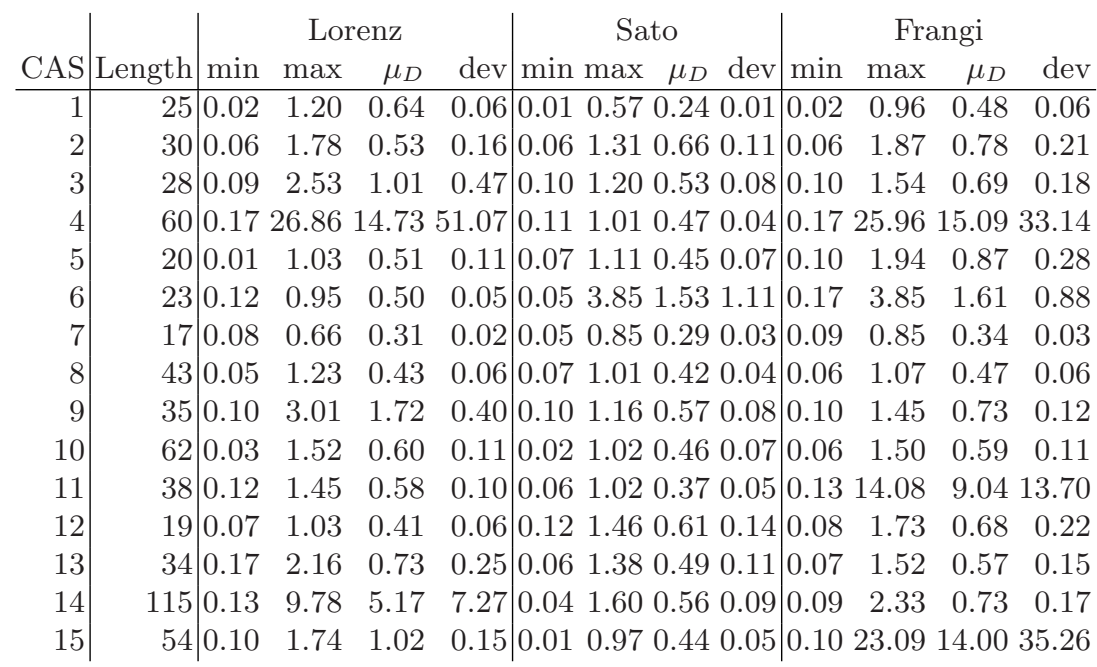

For the Sato filter, $\mu_{D}<2 \mathrm{~mm}$ for all CAS, with $D_{A R}(t) \in[0,1.6] \mathrm{mm}$ with the exception of one case. For the Lorenz filter, $\mu_{D}<2 \mathrm{~mm}$ for $13 \mathrm{CAS}$, with $D_{A R}(t) \in[0,3] \mathrm{mm}$. For the Frangi filter, $\mu_{D}<2 \mathrm{~mm}$ for $12 \mathrm{CAS}, D_{A R}(t) \in$ $[0,3.85] \mathrm{mm}$. Figure 2 illustrates the overall mean performance. The average results obtained on the basis of the Sato filter are best in 12 cases, while Lorenz was best in the remaining three (CAS 2, 6 and 12).

Figure [3-left illustrates a typical automatic path $\left(\mu_{D} \leq 0.85 \mathrm{~mm}\right.$, max distance $\approx 1 \mathrm{~mm})$. Note that staircase effect on the automatic path is responsible for most of the differences with respect to the reference. This is also observed for the majority of paths where the average distance is small. Figure 3-right illustrates an erroneous result obtained with the Frangi filter. In this case, the differences between the automatic path and the reference are due to a wrong trajectory induced by neighboring structures that also respond strongly to this filter.

\section{Discussion and Conclusions}

The results presented in Sect. 5] show that reasonable central axis tracking can be achieved for short coronary segments in most cases, adopting all filters, with 


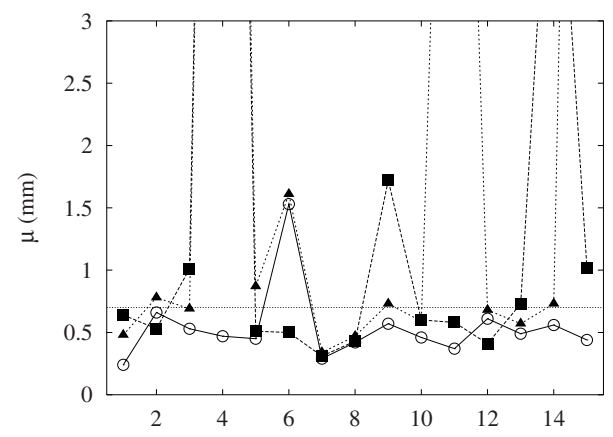

Fig. 2. Mean distance of automatic CVA obtained based on the Lorenz (squares), Sato (circles) and Frangi (triangles) filters. The horizontal line indicates $0.75 \mathrm{~mm}$. Mean errors larger than $3 \mathrm{~mm}$ are not shown.
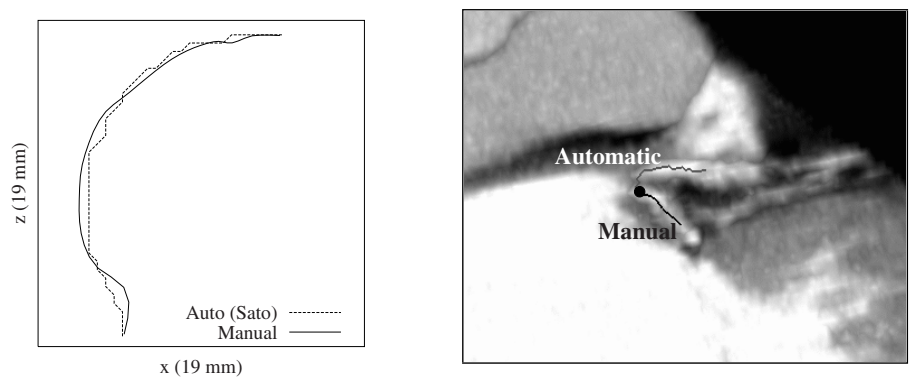

Fig. 3. Illustration of selected paths generated with the MCP method (projections where the differences between the automatic and reference paths are most visible). Left: Typical result (CAS 7, Sato filter). Right: Segment of erroneous result obtained with the Frangi filter (CAS 11) where another vessel is followed buy the MCP.

$\mu_{D}$ usually smaller than $1.5 \mathrm{~mm}$, which represents errors in the order of 1-2 voxels. In a few cases, specially with the Frangi filter, erroneous results were obtained due to a high filter response in the neighbourhood of the CVA, caused by a neighboring structure, and a low response near or at the CVA, caused by a decrease in intensity inside the vessel. In such situations, the MCP method is unable to follow the desired path, since it is "interrupted" by the low filter response. The Sato filter, however, seems to be less sensitive to this problem, with a more stable behaviour and smaller errors $(\mu \leq 0.85 \mathrm{~mm})$ in most CVAs. Note that the essential differences between the Sato and Frangi filters resides in the background noise elimination term $S$ (Eq.(4)), which could explain a weak response when the contrast is low.

The next step is to determine whether the error margin found in these experiments would be acceptable for using the CVAs tracked automatically in further 
processing, for example to generate cross-sectional and longitudinal views. Figure 4 illustrates the longitudinal views for $3 \mathrm{CAS}$ obtained with the manual and with the automatic CVAs. The differences are small, nearly unnoticeable. As for the cross-sectional view, the differences are larger. This is expected, since the staircase effect has large impact on the tangent along the path, which determines the orientation of the cross-sectional plane. Smoothing the automatic path could be a solution to reduce this problem.
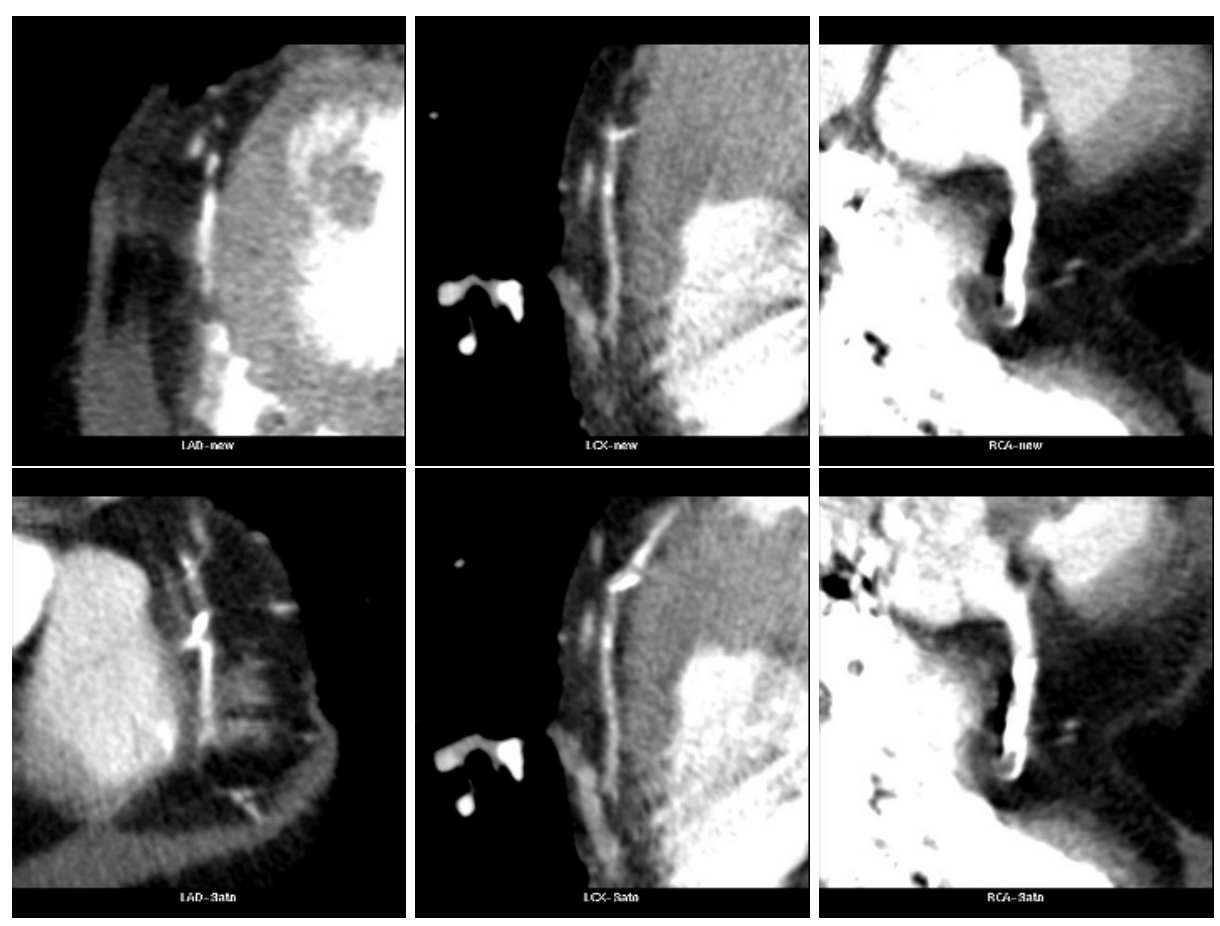

Fig. 4. Illustration of longitudinal views obtained with the EasyVision Visualization Tool for CAS 7,8,9 using the reference path (upper row) and the automatic path determined on the basis of the Sato filter (lower row). From left to right: LAD, LCX, RCA.

Finally, better results are expected for images acquired with 16-detector CT scanners. In a preliminary test with images recently acquired at our hospital (Philips scanner, $0.4 \mathrm{~mm}^{3}$ voxels), we could trace the CVA of the main coronary arteries (RCA, LAD, LCX) for longer trajectories as presented in Sect. 5 ( 85 mm, $74 \mathrm{~mm}, 28 \mathrm{~mm}$ ) with only two mouse clicks. The Sato filter was used to generate the cost function, with the same settings described in Sect. 2] In the 3 CAS, $D_{A R} \in[0,0.9]$ and $\mu_{D} \in[0.31,0.38]$, which is below the voxel size. Although a higher level of automation would be desired for the extraction of the complete 
coronary artery tree (e.g., [11]), we believe that our approach can be useful for cases where it is necessary to inspect a given segment of the CA, or when image quality is degraded by artifacts that cause the vessels to contain gaps or large discontinuities between slices.

Acknowledgements. We are grateful to the following institutions for providing the 4-detector CT data for this study: Univ. Hosp. Cleveland (USA); Diagnostico Maipu (Argentina); Klinik Mue-Pasing (Prof. Haberl, Germany) and Rad. Klinik Univ. Bonn (Germany). We also acknowledge the collaboration of radiologists in the University Medical Center Utrecht (Dr. E.J.Vonken and Dr. M. Prokop) for helping us with image interpretation and for providing the 16-detector dataset used in the study. This research is funded by Philips Medical Systems, Medical IT - Advanced Development, Best (NL).

\section{References}

1. S. Schroeder, A.F. Kopp, B. Ohnesorge, H. Loke-Gie, A. Kuettner, A. Baumbach, C. Herdeg, C.D. Claussen, and K.R. Karsch. Virtual coronary angioscopy using multislice computed tomography. Heart, 87(3):195-197, March 2002.

2. C.M. van Bemmel, L. Spreeuwers, M.A. Viergever, and W.J. Niessen. Level-set based carotid artery segmentation for stenosis grading. In MICCAI, pages 36-43, 2002.

3. O. Wink, W.J. Niessen, A.F. Frangi, B. Verdonk, and M.A. Viergever. 3D MRA coronary axis determination using a minimum cost approach. Magnetic Resonance in Medicine, 47(6):1169-1175, 2002.

4. S.R. Aylward and E. Bullit. Initialization, noise, singularities, and scale in height ridge traversal for tubular object centerline extraction. IEEE TMI, 21(2):61-75, 2002.

5. O. Wink, W.J. Niessen, B. Verdonk, and M.A. Viergever. Vessel axis determination using wave front propagation analysis. In MICCAI, pages 845-853, 2001.

6. O. Wink, W. J. Niessen, and M. A. Viergever. Minimum cost path determination using a simple heuristic function. In $I C P R, 2000$.

7. A.F. Frangi, W.J. Niessen, K.L. Vincken, and M.A. Viergever. Multiscale vessel enhancement filtering. In MICCAI, pages 130-137. Springer Verlag, 1998.

8. C. Lorenz, I.-C. Carlsen, T.M. Buzug, C. Fassnacht, and J. Weese. Multi-scale line segmentation with automatic estimation of width, contrast and tangential direction in 2d and 3d medical images. In CRVMed and MRCAS, pages 233-242, 1997.

9. Y. Sato, S. Nakajima, N. Shigara, H. Atsumi, T. Koller, G. Gerig, and R. Kikinis. Three-dimensional multi-scale line filter for segmentation and visualization of curvilinear structures in medical images. Med.Img.Analysis, 2(2):143-168, 1998.

10. S.D. Olabarriaga, M. Breeuwer, and W.J. Niessen. Evaluation of Hessian-based filters to enhance the axis of coronary arteries in $\mathrm{CT}$ images. In $C A R S$, pages 1191-1196. Elsevier, June 2003.

11. C. Lorenz, S. Reinisch, and T. Bullow T. Schlatholter. Simultaneous segmentation and tree reconstruction of the coronary arteries in msct images. In SPIE Medical Imaging, page 5031. SPIE, 2003. 